\title{
Múltiples índices espectrales para predecir la variabilidad de atributos estructurales y funcionales en zonas áridas
}

\author{
Mariana A. Buzzi ${ }^{12}$ 凹; ; B́́rbara L. Rueter ${ }^{1} \&$ Luciana Ghermandi $^{3}$ \\ ${ }^{1}$ Departamento de Biología y Ambiente. Facultad de Ciencias Naturales y de la Salud. UNPSJB. Comodoro Rivadavia, Chubut, \\ Argentina. ${ }^{2}$ Grupo de Estudios Biofísicos y Ecofisiológicos (GEBEF)-CONICET. ${ }^{3}$ Laboratorio Ecotono, Universidad Nacional \\ del Comahue, INIBIOMA-CONICET, Bariloche, Río Negro, Argentina.
}

\begin{abstract}
Resumen. En la región patagónica Argentina, las principales fuerzas directrices de la degradación son las actividades extractivas (petrolera y minera) y la ganadería ovina extensiva. Evaluar la capacidad de los índices espectrales para predecir la variabilidad espacial de los atributos estructurales y funcionales en ecosistemas áridos permite identificar patrones asociados a la desertificación o a la restauración pasiva. Uno de los principales problemas a sortear en estos ambientes es la gran extensión territorial. En este sentido, los índices espectrales provenientes de sensores remotos constituyen una herramienta útil para evaluar áreas extensas. El objetivo de este trabajo fue evaluar la capacidad de múltiples índices espectrales del satélite SPOT 5 para predecir la variabilidad espacial de un atributo estructural (cobertura vegetal de especies perennes) y un atributo funcional (productividad forrajera) en zonas áridas. Se evaluó la relación de siete índices espectrales obtenidos del satélite SPOT 5, en 39 sitios ubicados en tres unidades de paisaje denominadas cañadones costeros ( $n=7)$, pampas $(\mathrm{n}=18)$ y valles occidentales $(\mathrm{n}=14)$ de Patagonia central. El "green normalized difference vegetation index" (GNDVI), el "two modified soil adjusted vegetation index" (MSAVI2), el "normalized difference vegetation index" (NDVI) y el "ratio vegetation index" (RVI) fueron los mejores predictores de la cobertura vegetal de especies perennes en la escala de paisaje. Los índices espectrales explicaron más de $34 \%$ de la variación de la productividad forrajera en el área de estudio y más de $60 \%$ en los valles occidentales. Los resultados de este trabajo indican que los índices espectrales provenientes del satélite SPOT 5, principalmente el GNDVI, el MSAVI2, el NDVI y el RVI, constituyen herramientas apropiadas para predecir los cambios de la estructura y función de la vegetación a nivel de las unidades de paisaje de zonas áridas.
\end{abstract}

[Palabras clave: Patagonia árida, índices de vegetación, satélite SPOT 5]

\begin{abstract}
Aвsтract. Multiple spectral indices to predict the variability of structural and functional attributes in arid areas. In the Argentinean Patagonia, the main driving forces of degradation are the extractive activities (oil and mining) and sheep ranching. Determine the ability of spectral indices to predict the spatial variability of structural and functional attributes of arid ecosystems would help to identify patterns associated with desertification or passive restoration. One of the main problems in these environments is their large territorial extension. Thus, remote sensing indices could be useful tools for assessing these areas. The aim of this study was to evaluate the ability of multiple spectral indices SPOT 5 satellite to predict the spatial variability of a structural attribute (perennial vegetation cover) and of a functional attribute (forage production) in arid areas. We assessed the relationship of seven spectral indices obtained from the satellite SPOT 5 in 39 sites located in three landscape units: coastal valleys $(n=7)$, plateaus $(n=18)$ and eastern valleys $(n=18)$ in central Patagonia (Argentina). The green normalized difference vegetation index (GNDVI), the two modified soil adjusted vegetation index (MSAVI2), the ratio vegetation index (RVI) and the normalized difference vegetation index (NDVI) were the best predictors of perennial vegetation cover at landscape level. The spectral indices accounted for more than $34 \%$ of the variation in forage production in whole study area and for more than $60 \%$ in the western valleys. Our results show that spectral indices from satellite SPOT 5, mainly the GNDVI, the MSAVI2, the NDVI and the RVI, are appropriate tools to predict changes of the structure and function of the vegetation at landscape units level in arid zones.
\end{abstract}

[Keywords: arid Patagonia, vegetation indices, SPOT 5 satellite]

\section{INTRODUCCIÓN}

Las regiones áridas y semiáridas ocupan dos quintas partes de la superficie terrestre y sostienen la vida de un billón de personas (Reynolds et al. 2007). Las actividades extractivas, como las industrias minera y petrolera, y la ganadería ovina extensiva han generado desertificación en una vasta área de América del Sur, puntualmente en la región

Editor asociado: Esteban Jobbágy

marianabuzzi86@gmail.com patagónica (Soriano and Movia 1986; Ares et al. 1990; Soriano and Paruelo 1990; Golluscio et al. 1998; Paruelo and Aguiar 2003). Por medio de la metodología propuesta por la Food and Agriculture Organization (FAO) para evaluar la desertificación (FAO 1984), del Valle (1998) estimó que $35 \%$ de la región patagónica tiene una desertificación de media a severa, 23\% severa y $8 \%$ muy severa. 
Una característica de los ecosistemas de zonas áridas y de gran parte de las semiáridas de Patagonia es que la calidad forrajera no se vincula de forma lineal con la cobertura de especies, sino que es una combinación de diferentes formas biológicas (i.e., arbustos, subarbustos, dicotiledóneas herbáceas y pastos) y de distintas partes de plantas (i.e., tallos, hojas, flores y frutos). El método de valor pastoral ha sido muy utilizado a escala predial para determinar la calidad forrajera y la receptividad ganadera de los ecosistemas en Patagonia árida (Elissalde et al. 2002). Este método establece una relación entre la cobertura vegetal con la productividad forrajera a partir de incorporar el índice de calidad específica, que establece un rango de preferencia del ganado según la calidad nutricional de las plantas, la aptitud para ser utilizadas como forraje, la palatabilidad y el período factible de ser pastoreadas (Passera and Borseto 1983).

El estudio de los atributos estructurales y funcionales de los ecosistemas en áreas extensas, comolas zonas áridas y semiáridas del planeta, estuvo metodológicamente limitado hasta que los sensores remotos comenzaron a proveer de valiosa información espectral (Irisarri et al. 2012). Los índices espectrales son una herramienta que provee información rápida y de manera no destructiva (Running et al. 2000) en extensiones territoriales grandes con una resolución espacial moderada y temporal alta (Irisarri et al. 2012).

La misión del satélite SPOT 5 pertenece a una de las últimas generaciones de misiones SPOT con mejoras significativas en términos de los instrumentos de a bordo, del sistema autónomo de control de posicionamiento y altitud que permiten alta precisión de la localización absoluta (Riazanoff 2002). El satélite SPOT tiene una resolución temporal de 26 días, una órbita helio-sincrónica y las imágenes satelitales que generan se caracterizan por ser pancromáticas o multiespectrales, con una resolución de 2.5, 5 y 10 m, respectivamente, lo que permite la construcción de cartografía con excelente calidad geométrica.

Los índices espectrales pueden clasificarse en cuatro grupos: a) índices de vegetación simples (e.g., el "difference vegetation index" (DVI), el "normalized difference vegetation index" (NDVI) (Rouse et al. 1973) y el "ratio vegetation index" (RVI) [Jordan 1969]), b) índices de vegetación desarrollados para ajustarse a la influencia del suelo desnudo (e.g., el "soil adjusted vegetation index" [SAVI] [Huete 1988] y el "two modified soil adjusted vegetation index" [MSAVI2]), c) índices de vegetación que combinan correcciones para disminuir la influencia del suelo desnudo o los efectos atmosféricos (e.g., el "enhanced vegetation index" [EVI] [Huete et al. 2002] y el "two band enhanced vegetation index" [EVI2] [Jiang et al. 2008], y d) índices de vegetación estimados para incrementar la relación lineal entre el índice y los parámetros biofísicos (e.g., el "green normalized difference vegetative index" [GNDVI] [Gitelson et al. 1996]). Estudios recientes mostraron que el NDVI puede predecir de manera satisfactoria índices "landscape function analysis" (LFA) en minas restauradas de Australia (Ong et al. 2009 ), en pastizales semiáridos de España (García-Gómez and Maestre 2011) y en una vasta extensión de Patagonia $(800000$ km²) (Gaitán et al. 2013). No obstante, si se tiene en cuenta la herramienta que ofrece la resolución espacial del satélite SPOT 5, se planteó como objetivo de este trabajo evaluar la capacidad de los índices espectrales del satélite SPOT 5 para predecir la variabilidad espacial de la cobertura vegetal de especies perennes y la productividad forrajera en zonas áridas. Para cumplir con el objetivo pautado se realizaron dos preguntas: 1) ¿Pueden los índices espectrales predecir atributos en los ecosistemas áridos?,y 2) ¿Cuál de estos índices espectrales representan mejor el sistema?

\section{Materiales y MÉTOdOS}

\section{Área de estudio}

El área de estudio se encuentra en el sur-este de la provincia de Chubut, en la Patagonia argentina (Figura 1y 2). El 90\% de la Patagonia es semiárida y árida, excepto a lo largo de la Cordillera de los Andes, que se caracteriza por una biozona húmeda. Las precipitaciones en el área de estudio son $250 \mathrm{~mm}$ /año y presentan un régimen mediterráneo (se concentran en otoño-invierno). La temperatura media anual es $12.5{ }^{\circ} \mathrm{C}$ y dominan vientos fuertes con dirección oeste-este (Beeskow et al. 1987). El área de estudio se encuentra en la Provincia fitogeográfica Patagónica. Comprende los distritos Central y del Golfo San Jorge (Soriano 1956; Cabrera 1976; León et al. 1998). Se trabajó en las tres principales unidades de paisaje detectadas a escala regional (1: 250000): cañadones costeros $\left(463.65 \mathrm{~km}^{2}\right)$, pampas $\left(575.33 \mathrm{~km}^{2}\right)$ y valles occidentales $\left(161 \mathrm{~km}^{2}\right)$ (Buzzi et al. 2013). Los cañadones costeros están dominados por matorrales 


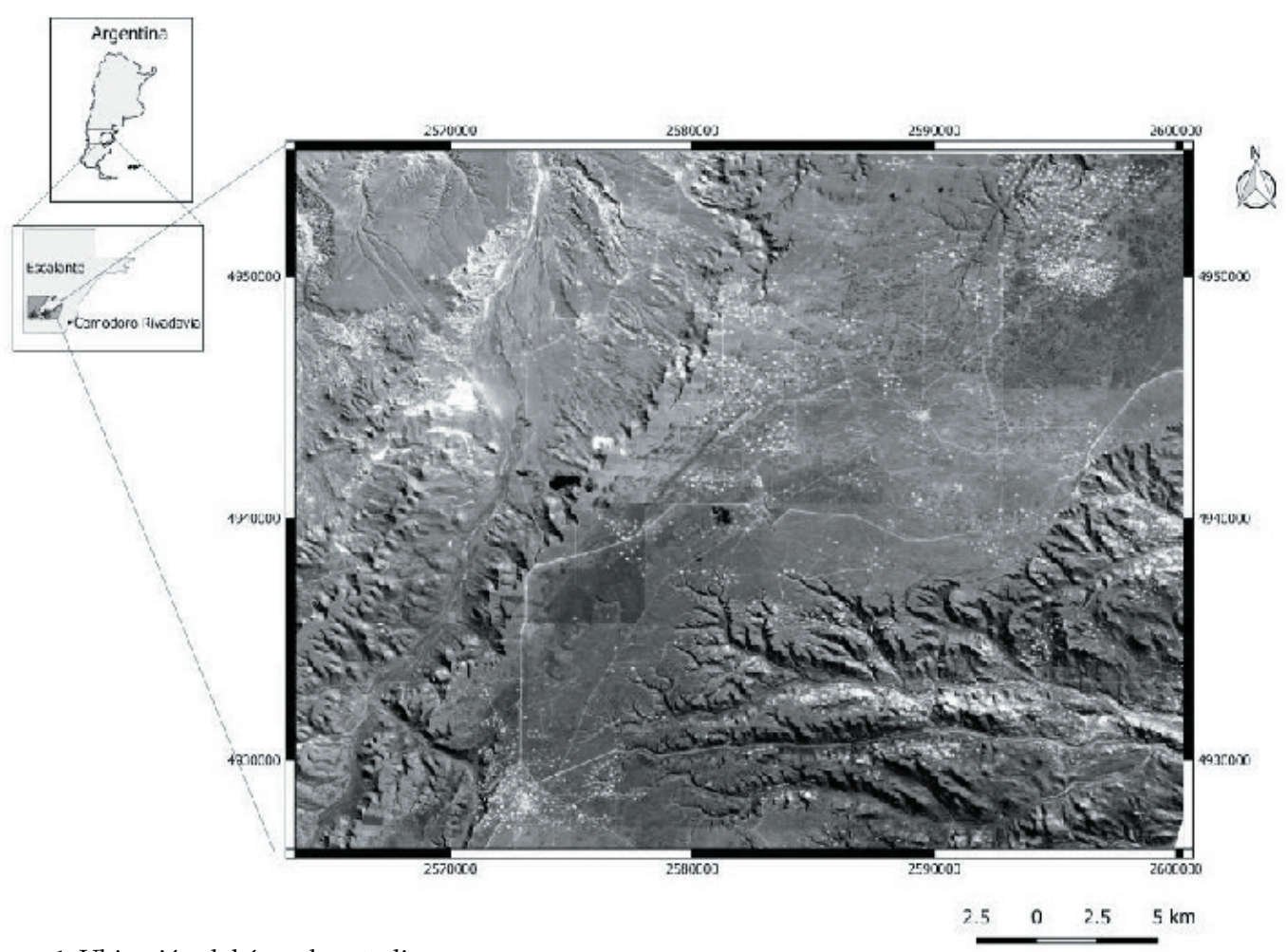

Figura 1. Ubicación del área de estudio.

Figure 1. Study site location.

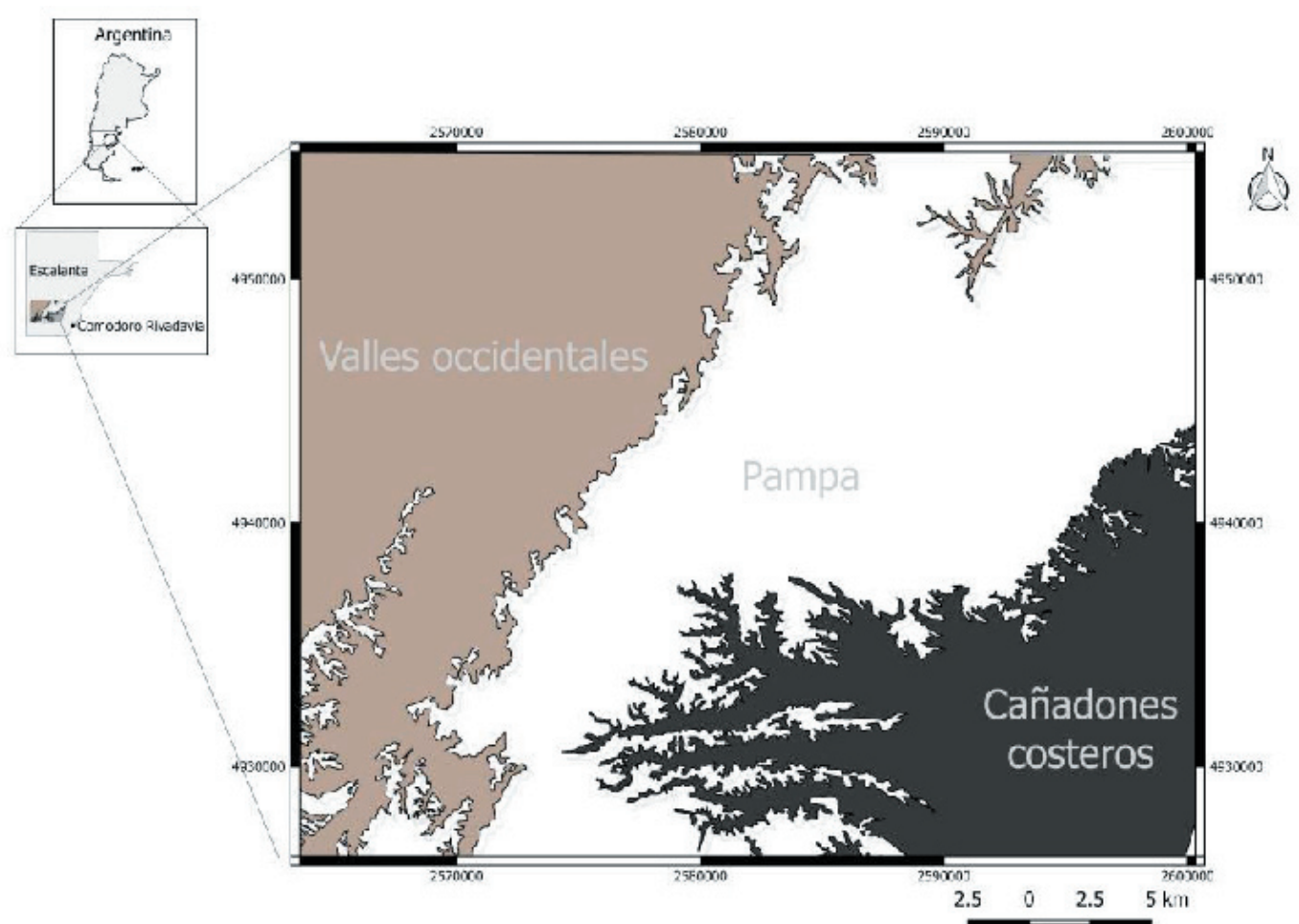

Figura 2. Unidades de paisaje.

Figure 2. Landscape units. 
abiertos y cerrados de Colliguaja integerrima Gillies and Hooky Retanilla patagonica (Speg.) Tortosa, en las porciones de mayor altura y estepas arbustivas en las proximidades al mar (Rueter and Bertolami 2010). Las pampas se caracterizan por ser estepas graminosas de Pappostipa humilis (Cav.) Romasch, Poa ligularis Nees ex Steud y Festuca argentina (Speg.) Parodi, con diferentes grados de conectividad entre sí. En los valles occidentales dominan las estepas subarbustivas de Nassauvia glomerulosa (Lag. ex Lindl.) D. Don, Nassauvia ulicina (Hook. f.) Macloskie y Chuquiraga aurea Skottsb, distribuidas de manera heterogénea en el espacio.

\section{Determinación de los datos espectrales}

Se trabajó con imágenes del satélite SPOT 5 de los meses de julio de 2013, febrero y septiembre de 2014. Las imágenes corresponden al sensor "Large Field High Resolution" (HRG), que posee una resolución espectral de cuatro bandas: XS1 Verde (0.495-0.605 $\mu \mathrm{m})$, XS2 Rojo (0.617-0.687 $\mu \mathrm{m})$, XS3 Infrarrojo Cercano $(0.780-0.893 \mu \mathrm{m})$ y SWIR Infrarrojo Medio $(1.545-1.750 \mu \mathrm{m})$. El satélite SPOT 5 tiene una resolución espacial de $10 \mathrm{~m}$ y temporal de 26 días. La calidad de la información está bajo el nivel de procesamiento 2A, la geometría coincide con una proyección y un sistema geodésico estándar (datum y elipsoide WGS84) (Riazanoff 2002). La calibración radiométrica es un pre-requisito para la obtención de datos de calidad generando productos derivados de alto nivel. Las imágenes fueron calibradas radiométricamente mediante el complemento "top of atmosphere reflectance" (TOA) del programa QGIS v. 2.6, que consiste en la conversión de los niveles digitales a valores de radiancia y posteriormente de reflectancia (32 bits) considerando los coeficientes de calibración disponibles para el satélite y las fechas utilizadas (Chander et al. 2009). Los valores de reflectancia fueron reescalados por el mismo complemento, de forma tal que los resultados obtenidos están en un rango entre 0 y 255. La información necesaria para la aplicación de este complemento fue tomada de las cabeceras de las imágenes (Paruelo et al. 2004). Los datos de reflectancia fueron usados para calcular siete de los índices más citados en la bibliografía, a partir de los valores medios obtenidos de las imágenes utilizadas (Tabla 1).

\section{Obtención de datos de campo}

Se trabajó en 39 sitios ubicados en las unidades de paisaje denominadas cañadones costeros $(n=7)$, pampas $(n=18)$ y valles occidentales ( $n=14)$ (Buzzi et al. 2013). Los sitios se ubicaron en áreas con actividad petrolera y ganadería ovina extensiva con cargas representativas de la región. Se trabajó en parcelas de 1 ha (100x100 m) ubicadas en áreas de homogeneidad vegetal para garantizar que la parcela muestreada sea representativa de la clase espectral provista por el sensor. Se evaluó la relación entre los índices espectrales del punto de muestreo a campo, correspondiente a una determinada clase de información, y el valor medio de una matriz de 10x10 pixeles en torno a dicho punto (clase espectral), a fin de verificar que el sitio de muestreo se correspondía con un área de características homogéneas. Se relevaron atributos estructurales y funcionales de la vegetación mediante el método "line intercept" (Canfield 1941), adaptado por Passera et al. (1986), Elissalde et al. (2002) y Bertolami et al. (2005). Las transectas realizadas tuvieron una longitud de $50 \mathrm{~m}$ y fueron orientadas en la dirección principal del flujo de los recursos (pendiente o dirección del viento). La cobertura se estimó basado en el supuesto teórico que la presencia de una especie es equivalente a su cobertura (Daget and Poissonet 1975; Greig-Smith 1983). La productividad forrajera se estimó con el método "point quadrat modificado" (Passera et al. 1986), para lo cual se utilizó una aguja de $1.5 \mathrm{~m}$ de longitud y $4 \mathrm{~mm}$ de ancho, y el valor pastoral, que sintetiza la capacidad forrajera de la comunidad vegetal y cuantifica su calidad a partir de la composición florística y del valor forrajero relativo de las especies presentes. A partir del valor pastoral y utilizando factores de correlación se obtuvo la productividad forrajera expresada en $\mathrm{kg}$ de materia seca por hectárea por año $\left(\mathrm{kg} \mathrm{MS} \cdot \mathrm{ha}^{-1} \cdot \mathrm{añno}^{-1}\right)$.

\section{Análisis de los datos}

Se utilizaron regresiones lineales para evaluar la relación existente entre la cobertura vegetal de especies perennes y la productividad forrajera con los índices espectrales, a nivel de paisaje y de las unidades de paisaje.

\section{Resultados}

\section{Atributos estructurales y funcionales}

Los cañadones costeros presentaron una cobertura media de especies perennes de $61 \%$; las pampas, de $58 \%$ y los valles occidentales de $43 \%$. La productividad forrajera, estimada en las mismas unidades de paisaje, fue de 16, 15.4 y 11.83 kg MS.ha- ${ }^{-1}$.año ${ }^{-1}$, respectivamente (Tablas 2 a 4 ). 
Tabla 1. Índices espectrales del satélite SPOT 5 utilizados en este trabajo.

Table 1. Spectral indices from SPOT 5 satellite used in this work.

\begin{tabular}{|c|c|c|c|}
\hline Índice & Ecuación & Constante & Autores \\
\hline $\begin{array}{c}\text { NDVI } \\
\text { (normalized difference vegetation index) }\end{array}$ & $\frac{\rho N I R-\rho R}{\rho N I R+\rho R}$ & No posee & $\begin{array}{l}\text { Rouse et al. } \\
\quad(1973)\end{array}$ \\
\hline $\begin{array}{c}\text { GNDVI } \\
\text { (green NDVI) }\end{array}$ & $\frac{\rho N I R-\rho G}{\rho N I R+\rho G}$ & No posee & $\begin{array}{l}\text { Gitelson et al. } \\
\quad \text { (1996) }\end{array}$ \\
\hline $\begin{array}{c}\text { RVI } \\
\text { (ratio vegetation index) }\end{array}$ & $\frac{\rho N I R}{\rho R}$ & No posee & $\begin{array}{l}\text { Jordan } \\
(1969)\end{array}$ \\
\hline $\begin{array}{c}\text { DVI } \\
\text { (difference vegetation index) }\end{array}$ & $\rho N I R-\rho R$ & No posee & $\begin{array}{l}\text { Tucker } \\
(1979)\end{array}$ \\
\hline $\begin{array}{c}\text { EVI2 } \\
\text { (two-band enhanced vegetation index) }\end{array}$ & $G \times \frac{(\rho N I R-\rho R)}{(\rho N I R+C \times \rho R)+L}$ & $\begin{array}{c}\mathrm{G}=2.5 \\
\mathrm{~L}=1 \\
\mathrm{C}=2.4\end{array}$ & $\begin{array}{l}\text { Jiang et al. } \\
\text { (2008) }\end{array}$ \\
\hline $\begin{array}{c}\text { SAVI } \\
\text { Soil Adjusted Vegetation Index }\end{array}$ & $\frac{\rho N I R-\rho R}{(\rho N I R+\rho R+L) \times(1+L)}$ & $\mathrm{L}=0.5$ & $\begin{array}{c}\text { Huete ( } \\
\text { 1988) }\end{array}$ \\
\hline $\begin{array}{c}\text { MSAVI2 } \\
\text { (modified soil adjusted vegetation index) }\end{array}$ & $\frac{2 \times \rho N I R+1-\sqrt{(2 \times \rho N I R+1)^{2}-8(\rho N I R-\rho R)}}{2}$ & No posee & $\begin{array}{l}\text { Qi et al. } \\
(1994)\end{array}$ \\
\hline
\end{tabular}

$\rho N I R, \rho R$ y $\rho$ Gson la reflectancia del infrarrojo cercano, rojo y verde, respectivamente.

\begin{tabular}{ccccc}
\hline \multicolumn{5}{c}{ Cañadones Costeros } \\
\hline Variable & Media & Desvío estándar & Mínimo & Máximo \\
CV $(\%)$ & 61.142 & 18.370 & 35 & 85 \\
PF $\left(\mathrm{kg} \mathrm{MS.ha}^{-1} \cdot \mathrm{año}^{-1}\right)$ & 16.005 & 17.819 & 4 & 65 \\
NDVI & 0.133 & 0.055 & 0.023 & 0.201 \\
GNDVI & 0.119 & 0.089 & 0.103 & 0.213 \\
RVI & 1.327 & 0.137 & 1.090 & 1.507 \\
DVI & 0.040 & 0.018 & 0.070 & 0.013 \\
EVI2 & 0.073 & 0.013 & 0.060 & 0.098 \\
SAVI & 0.075 & 0.030 & 0.024 & 0.123 \\
MSAVI2 & 0.475 & 0.067 & 0.413 & 0.608 \\
\hline
\end{tabular}

CV: cobertura vegetal de especies perennes; PF: productividad forrajera.

\begin{tabular}{ccccc}
\hline & \multicolumn{3}{c}{ Pampa } \\
\hline Variable & Media & Desvío estándar & Mínimo & Máximo \\
CV $(\%)$ & 57.842 & 15.532 & 27 & 88 \\
PF $\left(\mathrm{kg} \mathrm{MS}^{-1} \mathrm{ha}^{-1} \cdot \mathrm{año}^{-1}\right)$ & 15.407 & 7.620 & 2.730 & 31.350 \\
NDVI & 0.120 & 0.030 & 0.096 & 0.217 \\
GNDVI & 0.128 & 0.032 & 0.083 & 0.227 \\
RVI & 1.324 & 0.088 & 1.213 & 1.572 \\
DVI & 0.038 & 0.010 & 0.027 & 0.070 \\
EVI2 & 0.069 & 0.009 & 0.045 & 0.081 \\
SAVI & 0.073 & 0.017 & 0.052 & 0.128 \\
MSAVI2 & 0.451 & 0.078 & 0.340 & 0.651 \\
\hline
\end{tabular}

Tabla 2. Valores medios, desvíos estándares, máximos y mínimos de las variables estructurales y funcionales e índices espectrales en los cañadones costeros.

Table 2. Mean values, standard deviations, maximum and minimum values of structural and functional variables and spectral indices in coastal valleys.

$\mathrm{CV}$ : cobertura vegetal de especies perennes; PF: productividad forrajera.

\begin{tabular}{ccccc}
\hline \multicolumn{5}{c}{ Valles occidentales } \\
\hline Variable & Media & Desvío estándar & Mínimo & Máximo \\
CV $(\%)$ & 43.200 & 28.847 & 5 & 82 \\
PF $\left(\mathrm{kg} \mathrm{MS.ha}^{-1} \cdot \mathrm{anno}^{-1}\right)$ & 11.83 & 9.027 & 0 & 29.110 \\
NDVI & 0.098 & 0.028 & 0.062 & 0.158 \\
GNDVI & 0.111 & 0.026 & 0.053 & 0.151 \\
RVI & 1.219 & 0.028 & 0.062 & 0.158 \\
DVI & 0.032 & 0.008 & 0.020 & 0.042 \\
EVI2 & 0.055 & 0.014 & 0.035 & 0.079 \\
SAVI & 0.057 & 0.013 & 0.038 & 0.080 \\
MSAVI2 & 0.295 & 0.105 & 0.056 & 0.415 \\
\hline
\end{tabular}

Tabla 3. Valores medios, desvíos estándares, máximos y mínimos valores entre variables estructurales y funcionales e índices espectrales en la pampa.

Table 3. Mean values, standard deviations, maximum and minimum values between structural and functional variables and spectral indices in plateau.

$\mathrm{CV}$ : cobertura vegetal de especies perennes; PF: productividad forrajera. 


\section{Relación índices espectrales, cobertura vegetal y productividad forrajera}

Los valores de los índices espectrales en los sitios de muestreo presentaron buenas relaciones con los valores medios de los índices calculados a partir de una matriz de 10x10 pixeles centrada en cada sitio (Anexo I, Información suplementaria). En la escala de paisaje, todos los índices espectrales analizados presentaron relaciones elevadas y significativas $(P<0.05)$ con la cobertura vegetal de especies perennes. La menor relación la mostró el DVI. A nivel de las unidades de paisaje, el GNDVI y el MSAVI2 mostraron buenas performances en los valles occidentales (Tabla 5). La productividad forrajera presentó relación positiva y significativa con todos los índices espectrales a nivel de paisaje. En los valles occidentales todos los índices explicaron más del $60 \%$ de la variación de la productividad forrajera (Tabla 6).

\begin{tabular}{|c|c|c|c|c|c|c|c|}
\hline \multicolumn{8}{|c|}{ En la escala de paisaje } \\
\hline & NDVI & GNDVI & RVI & DVI & EVI2 & SAVI & MSAVI2 \\
\hline $\mathrm{r}^{2}$ & $0.48^{*}$ & $0.52^{*}$ & $0.47^{*}$ & 0.29 & $0.40^{*}$ & $0.42^{*}$ & $0.52^{*}$ \\
\hline$P$ & 0.002 & 0.001 & 0.003 & 0.074 & 0.012 & 0.010 & 0.001 \\
\hline \multicolumn{8}{|c|}{ Cañadones costeros } \\
\hline & NDVI & GNDVI & RVI & DVI & EVI2 & SAVI & MSAVI2 \\
\hline $\mathrm{r}^{2}$ & 0.80 & 0.53 & 0.76 & 0.73 & 0.38 & 0.36 & 0.35 \\
\hline$P$ & 0.102 & 0.361 & 0.130 & 0.157 & 0.519 & 0.541 & 0.561 \\
\hline \multicolumn{8}{|c|}{ Pampas } \\
\hline & NDVI & GNDVI & RVI & DVI & EVI2 & SAVI & MSAVI2 \\
\hline $\mathrm{r}^{2}$ & 0.35 & 0.13 & 0.38 & 0.04 & 0.02 & 0.02 & 0.02 \\
\hline$P$ & 0.168 & 0.601 & 0.128 & 0.859 & 0.915 & 0.916 & 0.992 \\
\hline \multicolumn{8}{|c|}{ Valles occidentales } \\
\hline & NDVI & GNDVI & RVI & DVI & EVI2 & SAVI & MSAVI2 \\
\hline $\mathrm{r}^{2}$ & 0.48 & $0.54^{*}$ & 0.47 & 0.09 & 0.29 & 0.31 & $0.54^{*}$ \\
\hline$P$ & 0.081 & 0.044 & 0.086 & 0.759 & 0.321 & 0.290 & 0.044 \\
\hline
\end{tabular}

* Valores significativos $P<0.05$

\begin{tabular}{|c|c|c|c|c|c|c|c|}
\hline \multicolumn{8}{|c|}{ En la escala de paisaje } \\
\hline & NDVI & GNDVI & RVI & DVI & EVI2 & SAVI & MSAVI2 \\
\hline $\mathrm{r}^{2}$ & $0.40^{*}$ & $0.43^{*}$ & $0.38^{*}$ & $0.34^{*}$ & $0.39 *$ & $0.40^{*}$ & $0.38^{*}$ \\
\hline$P$ & 0.014 & 0.007 & 0.018 & 0.039 & 0.016 & 0.014 & 0.020 \\
\hline \multicolumn{8}{|c|}{ Cañadones costeros } \\
\hline & NDVI & GNDVI & RVI & DVI & EVI2 & SAVI & MSAVI2 \\
\hline $\mathrm{r}^{2}$ & 0.23 & 0.30 & 0.15 & 0.05 & 0.09 & 0.11 & 0.35 \\
\hline$P$ & 0.704 & 0.610 & 0.805 & 0.924 & 0.878 & 0.858 & 0.561 \\
\hline \multicolumn{8}{|c|}{ Pampas } \\
\hline & NDVI & GNDVI & RVI & DVI & EVI2 & SAVI & MSAVI2 \\
\hline$r^{2}$ & 0.17 & 0.12 & 0.10 & 0.16 & 0.14 & 0.14 & 0.20 \\
\hline$P$ & 0.512 & 0.645 & 0.675 & 0.527 & 0.583 & 0.590 & 0.437 \\
\hline \multicolumn{8}{|c|}{ Valles occidentales } \\
\hline & NDVI & GNDVI & RVI & DVI & EVI2 & SAVI & MSAVI2 \\
\hline $\mathrm{r}^{2}$ & $0.66^{*}$ & $0.71^{*}$ & $0.65^{*}$ & $0.61^{*}$ & $0.73^{*}$ & $0.73^{*}$ & $0.57^{*}$ \\
\hline$P$ & 0.010 & 0.004 & 0.011 & 0.020 & 0.003 & 0.003 & 0.033 \\
\hline
\end{tabular}

Tabla 5. Resumen de los modelos de regresión entre la cobertura de especies perennes y los índices espectrales a escala de paisaje y de unidades de paisaje.

Table 5. Summary of regression models between the cover of perennial species and spectral indices at landscape and landscape units level.
En este trabajo se compararon diferentes índices espectrales del satélite SPOT 5 para detectar variaciones espaciales en atributos . espectrales reflejaron el gradiente de aridez que va en aumento en el área de estudio, los cañadones costeros al este, hacia los presentaron los valores más elevados al este y fueron decreciendo a medida que disminuía el efecto costero sobre los ecosistemas.

habilidad de los índices espectrales para de cada uno. El MSAVI2 y el GNDVI fueron los mejores predictores de la cobertura vegetal de especies perennes en la escala de paisaje, lo que demuestra que tanto los ecosistémicas radica en las particularidades

* Valores significativos $P<0.05$ 
índices ajustados al suelo como aquellos diseñados para incrementar la relación lineal entre el índice y los parámetros biofísicos son buenos predictores de la cobertura vegetal. El SAVI muestra una ligera sensibilidad a las influencias del suelo al contar con un factor de corrección L que reduce el efecto del brillo. Huete 1988 sugiere utilizar el valor óptimo 0.5 para valores intermedios de cobertura vegetal. Sin embargo, y en coincidencia con Gaitán et al. (2013), este supuesto no fue apropiado en el área de estudio ya que el MSAVI2 elimina estas influencias por completo (Qi et al. 1994) y es mejor predictor de la cobertura de especies perennes en la escala de paisaje y en las unidades de paisaje.

El NDVI fue postulado como el mejor predictor de las variables estructurales como la cobertura y la riqueza de especies en los ecosistemas áridos, semiáridos y subhúmedos de Patagonia (Gaitán et al. 2013). Esto se corresponde con los datos obtenidos a nivel de paisaje en el área de estudio.

En la escala de paisaje, todos los índices mostraron una relación significativa superior a $34 \%$ con la productividad forrajera. En particular, el GNDVI demostró ser un buen predictor ya que es el índice menos afectado por la saturación de la reflectancia de la vegetación. Esto coincide con Gianelle et al. (2009). Este índice podría ser potencialmente utilizado para predecir los cambios en la capacidad productiva de las comunidades vegetales. En los valles occidentales, todos los índices mostraron una alta capacidad para predecir la variabilidad de la productividad forrajera.

La complejidad ambiental en zonas áridas requiere del uso de múltiples índices espectrales, como los presentados en este trabajo, para detectar cambios en la cobertura vegetal y calidad productiva. Así mismo se muestra la capacidad de los índices espectrales obtenidos de imágenes del satélite SPOT 5 para evaluar la variabilidad estructural y funcional a nivel de paisaje y de las unidades de paisaje en zonas áridas.

Agradecimientos. Este trabajo fue financiado parcialmente por la Comisión Nacional de Actividades Espaciales (CONAE) y por la Secretaria de Ciencia y Técnica de la Universidad Nacional de la Patagonia San Juan Bosco (UNPSJB) en el marco del PICT 005/10 “Monitoreo de las Unidades de Paisaje del Golfo San Jorge mediante técnicas de Teledetección". Los autores agradecen al Grupo de Estudios Biofísicos y Ecofisiológicos (GEBEF) por su colaboración.

\section{REFERENCIAS}

Ares, J. O., A. M. Bertiller, C. M. Rostagno, M. P. Irisarri, J. Anchorena, et al. 1990. Structural and dynamic characteristics of overgazed grasslands of northern Patagonia, Argentina. Pp. 149-175 in: A. Breymeyer (ed.). Managed grasslands: Regional Studies.Elsevier, Amsterdam.

Beeskow, A. M., H. Del Valle, and C. M. Rostagno. 1987. Los Sistemas Fisiográficos de la Región Árida y Semiárida de la Provincia del Chubut. SECYT. Puerto Madryn, Chubut, Argentina. PP. 143.

Bertolami, M. A., B. L. Rueter, and M. E. Benítez. 2005. Estimación de la producción forrajera a partir de valores pastoral en el sudeste del Chubut-Argentina. Multequina 14:29-38.

Bulcock, H. H., and G. P. Jewitt. 2010. Spatial mapping of leaf area index using hyper-spectral remote sensing for hydrological applications with a particular focus on canopy interception. Hydrol Earth Syst Sci 14:383-392, doi: 10.5194/hess-14-383-2010.

Buzzi, M., M. A. Bertolami, and B. L. Rueter. 2013. Delimitación de unidades de paisaje en Patagonia Central. Revista de la Asociación de Ecología de Paisaje 4(2):211-218.

Cabrera, A. L. 1976. Regiones fitogeográficas argentinas, Enciclopedia Argentina de Agricultura y Jardinería (2da ed.), Tomo II, Fase 1 ACME, Buenos Aires. Pp. 85.

Canfield, M. H. 1941. Application of the line interception method in sampling of range vegetation. J Arid Environ 39: 388-394.

Chander, G., B. L. Markham, and D. L. Helder. 2009. Summary of current radiometric calibration for Landsat MSS, TM, ETM+, and EO-1 ALI sensors. Remote Sens Environ 113:893-903, doi: 10.1016/j.rse.2009.01.007.

Daget, P., and J. Poissonet. 1971. Une méthoded'analyzephytologique des praieries; criteresd'application. Annales Aggonomiques 22(1):5-41.

Del Valle, H. F. 1998. Patagonian soils:a regional synthesis. Ecología Austral 8:103-123

Elissalde, N., J. M. Escobar, and V. B. Nakamatsu. 2002. Inventario y evaluación de pastizales naturales de la zona árida y semiárida de la Patagonia. Programa de Acción de Lucha contra la Desertificación. Convenio SDSyPA-INTAGTZ. Trelew.

FAO. 1984. Metodología provisional para la evaluación y la representación cartográfica de la desertización. FAOPNUMA, Roma. Pp. 74.

Gaitán, J. J., D. Bran, G. Oliva, G. Ciari, V. Nakamatsu, J. Salomone, et al. 2013. Evaluating the performance of multiple remote sensing indices to predict the spatial variability of ecosystem structure and functioning in Patagonian steppes. Ecol Indic 34:181-191, doi: 10.1016/j.ecolind.2013.05.007. 
García-Gómez, M., and F. T. Maestre. 2011. Remote sensing data predict indicators of soil functioning in semi-arid steppes, central Spain. Ecol Indic 11:1476-1481, doi: 10.1016/j.ecolind.2011.02.015.

Gitelson, A., Y. J. Kaufman, and M. N. Merzlyak. 1996. Use of a green channel in remote sensing of global vegetation from EOS-MODIS. Remote Sens Environ 58(3):289-298, doi:10.1016/S0034-4257(96)00072-7.

Golluscio, R. A., V. Deregibus, and J. M. Paruelo. 1998. Sustainability and range management in the Patagonian steppe. Ecología Austral 8:265-284.

Greig-Smith, P. 1983. Quantitative plant ecology. 3rd ed. Blackwell.

Hofmann, F., M. Köpen, F. Klawonn, and R. Roy. 2006. Unsupervised Pixel Clustering in Multispectral Images by Genetic Programming II. Pp. 37-52 in: Springer Science and Business Media Editor (ed.). Soft computing: Methodologies and applications. Vol. 32.

Huete, A., K. Didan, T. Miura, and E. Rodríguez. 2002. Overview of the radiometric and biophysical performance of the MODIS vegetation indices (Special Issue). Remote Sens Environ 83:195-213, doi: 10.1016/S0034-4257(02)00096-2.

Huete, A. R., and R. D. Jackson. 1987. Suitability of spectral indices for evaluating vegetation characteristics on arid environments. Remote Sens Environ 23:213-232, doi: 10.1016/0034-4257(87)90038-1.

Huete, A. R. 1988. A soil-adjusted vegetation index (SAVI). Remote Sens Environ 25:295-309, doi:10.1016/00344257(88)90106-X

Huete, A. R., G. Hua, J. Qu, A. Chebouni, and W. J. Van Leeuwen. 1992. Normalization of multidirectional red and NIR reflectances with the SAVI, Remote Sens Environ 40:1-20, doi: 10.1016/0034-4257(92)90074-T.

Irisarri, J. G. N., M. Oesterheld, J. M. Paruelo, and M. Texeira. 2012. Patterns and controls of aboveground net primary production in meadows of Patagonia. A remote sensing approach. J Veg Sci 23(1):114-126, doi: 10.1111/j.16541103.2011.01326.x.

Ji, L., L. Zhang, J. Rover, B. K. Wylie, and X. Chene. 2014. Geostatistical estimation of signal-to-noise ratios for spectral vegetation indices. ISPRS J Photogra Remote Sens 96:20-27, doi: 10.1016/j.isprsjprs.2014.06.013.

Jiang, Z., A. R. Huete, K. Didan, and T. Miura. 2008. Development of a two-band enhanced vegetation index without a blue band. Remote Sens Environ 112(10):3833-3845, doi: 10.1016/j.rse.2008.06.006.

Jordan, C. F. 1969. Derivation of leaf-area index from quality of light on the forest floor. Ecology, 50(4):663-666, doi: $10.2307 / 1936256$.

León, R. J. C., D. Bran, M. Collantes, J. M. Paruelo, and A. Soriano. 1998. Grandes unidades de vegetación de la Patagonia extra andina. Ecología Austral 8:125-144.

Ong, C., D. Tongway, M. Kacctta, and N. Hindly. 2009. Phase 1: Deriving Ecosystem function analysiz indices from airborne hyperexpectral data. CSIRO Exploration and Mining.

Paruelo, J. M., and M. Aguiar. 2003. El impacto humano sobre los ecosistemas: el caso de la desertificación. Ciencia Hoy 13:48-59.

Paruelo, J. M., R. A. Golluscio, J. P. Guerschman, A. Cesa, V. V. Jouve, and M. F. Garbulsky. 2004. Regional scale relationships between ecosystem structure and functioning: the case of the Patagonian steppes. Global Ecol Biogeogr 13:385-395, doi: 10.1111/j.1466-822X.2004.00118.x.

Passera, C. B., and O. Borsetto. 1983. Determinación del índice de calidad específico. Actas del Taller de Arbustos Forrajeros. Grupo regional FAO-IADIZA. Mendoza.

Passera, C. B., A. D. Dalmaso, and O. Borseto. 1986. Método de "Point Quadrat" modificado. Actas del Taller de Arbustos Forrajeros. Grupo regional FAO-IADIZA. Mendoza.

Qi, J., A. Chehbouni, A. R. Huete, Y. H. Kerr, and S. Sorooshian. 1994. A modified soil adjusted vegetation index. Remote Sens Environ 48(2):119-126, doi: 10.1016/0034-4257(94)90134-1.

Reynolds, J. F., D. M. Stafford Smith, E. F. Lambin, I. I. Turner, M. Mortimore, S. P. J. Batterbury, et al. 2007. Global desertification building a science for dryland development. Science 316:847-851.

Riazanoff, S. 2002. SPOT satellite geometry handbook. S-NT-73-12-SI.Edition 1 Revision 0. GAEL Consultant. Toulouse, France.

Rouse, J. W., R. H. Haas, J. A. Schell, and D. W. Deering. 1973. Monitoring vegetation systems in the great plains with ERTS. In: Third ERTS Symposium, NASA SP-351 I. Pp. 309-317.

Rueter, B. L., and M. A. Bertolami. 2010. Comunidades vegetales y factores ambientales en los cañadones costeros de Patagonia. Ecología Austral 20:17-25.

Rueter, B. L. 2013. Tesis doctoral, Aspectos estructurales y funcionales de las comunidades vegetales nativas de los Cañadones Costeros del Distrito del Golfo San Jorge. Su dinámica temporal. Universidad Nacional de la Patagonia San Juan Bosco. Comodoro Rivadavia, Argentina.

Running, S. W., P. E. Thornton, R. R. Nemani, and J. M. Glassy. 2000. Global terrestrial gross and net primary productivity from the Earth Observing System. Pp. 44-57 in: O. E. Sala, R. B. Jackson, H. A. Mooney and R. W. Howarth (eds.). Methods in ecosystem science,. Springer-Verlag, New York, USA.

Sims, N. C., and M. J. Colloff. 2012. Remote sensing of vegetation responses to flooding of a semi-arid floodplain: Implications for monitoring ecological effects of environmental flows. Ecol Indic 18:387-391, doi: 10.1016/ j.ecolind.2011.12.007.

Soriano, A., and J. M. Paruelo. 1990. El manejo de campos de pastoreo en Patagonia: Aplicación de principios ecológicos. Ciencia Hoy 2(7):44-53.

Soriano, A., and C. Movia. 1986. Erosión y desertización en la Patagonia. Interciencia 11:77-83.

Soriano, A. 1956. Los distritos florísticos de la provincia Patagónica. Rev Invest Agric 10:323-347.

Tucker, C. J. 1979. Red and Photographic Infrared Linear Combinations for Monitoring Vegetation. Remote Sens Environ 8:127-150, doi: 10.1016/0034-4257(79)90013-0. 